isotype control antibody. Furthermore, itgb $5^{-/-}$mice had significantly less a-SMA around their airways than wild type control mice in response to Asp.f treatment. However, both itgb5 $5^{-/-}$andantiaVß5 treated mice had significantly more airway inflammation and more inflammatory cells present in the bronchoalveolar lavage compared with their matched controls. These data provide evidence that airway smooth muscle cells can activate TGF- $B$ in vivo. Inhibition of, or genetic loss of, the aVß5 integrin significantly reduces allergen-induced increases in airway smooth muscle mass, however, peribronchial inflammation is increased consistent with the known effects of TGF $\beta$. Targeted inhibition of the aVß5 integrin may reduce airway remodelling, but global inhibition is unlikely to be useful due to the enhanced inflammatory response.

\section{S33 VITAMIN D AND AIRWAY REMODELLING IN PAEDIATRIC SEVERE THERAPY RESISTANT ASTHMA}

doi:10.1136/thoraxjnl-2011-201054b.33

${ }^{1} \mathrm{~A}$ Sjoukes, ${ }^{1} \mathrm{~A}$ Gupta, ${ }^{2} \mathrm{~T}$ Oates, ${ }^{1} \mathrm{~A}$ Bush, ${ }^{1} \mathrm{~S}$ Saglani. ${ }^{1}$ Department of Respiratory Paediatrics, Royal Brompton Hospital, London, UK; ${ }^{2}$ National Heart \& Lung Institute, Imperial College, London, UK

Background Serum vitamin D levels have been related to asthma control and medication use in children with mild to moderate disease, but little is known about the relationship between serum vitamin $\mathrm{D}$ levels and airway remodelling and mucosal inflammation in asthma. We hypothesised that lower serum vitamin $\mathrm{D}$ levels would be associated with increased airway inflammation and remodelling and lower lung function in children with severe therapy resistant asthma (STRA).

Methods Nineteen children aged between 6 and 16 years with STRA underwent spirometry, fiberoptic bronchoscopy, endobronchial biopsy, and measurement of serum vitamin $\mathrm{D}\left(25(\mathrm{OH}) \mathrm{D}_{3} \mathrm{nmol} / \mathrm{l}\right)$. Endobronchial biopsies stained with $\mathrm{H} \& \mathrm{E}$ were used to quantify airway remodelling (reticular basement membrane thickness, smooth muscle mass and epithelial shedding). Immunohistochemistry was used to quantify smooth muscle cell proliferation using proliferating cell nuclear antigen, and inflammatory cells (eosinophils, neutrophils and mast cells).

Results Seventeen of 19 children with STRA were vitamin D insufficient $(<50 \mathrm{nmol} / \mathrm{l})$, median (range) serum $25(\mathrm{OH}) \mathrm{D}_{3} \quad 29$ $(21-39) \mathrm{nmol} / \mathrm{l}$. There was no relationship between serum $25(\mathrm{OH})$ $\mathrm{D}_{3}$ and submucosal eosinophils, neutrophils or mast cells. Airway smooth muscle mass was inversely related to serum $25(\mathrm{OH}) \mathrm{D}_{3}$ $(\mathrm{r}=-0.6, \mathrm{p}=0.007)$ (Abstract S33 figure 1), but there was no relationship between vitamin $\mathrm{D}$ levels and reticular basement membrane thickness or epithelial shedding. Lung function was not related to

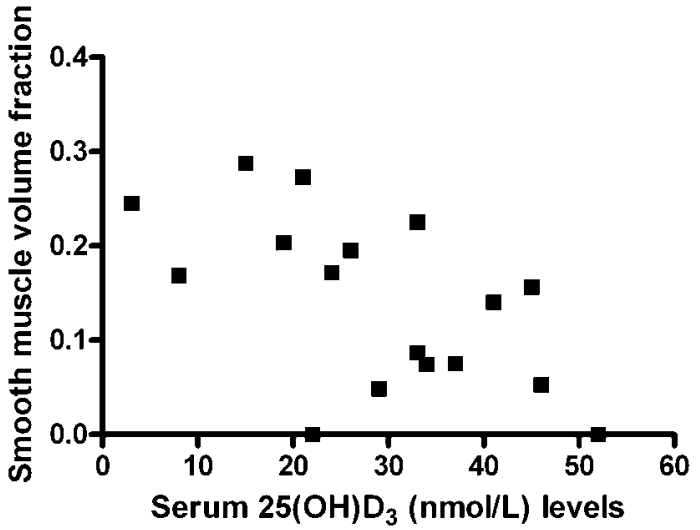

Abstract S33 Figure 1 Correlation between volume fraction of airway smooth muscle and serum $25(\mathrm{OH}) \mathrm{D}_{3}$ in paediatric STRA. serum vitamin $\mathrm{D}$ levels, however bronchodilator reversibility was inversely related to serum $25(\mathrm{OH}) \mathrm{D}_{3}$ levels $(\mathrm{r}=-0.53, \mathrm{p}=0.02)$.

Conclusions Vitamin D insufficiency is common in children with STRA. Lower vitamin D levels in children with STRA were associated with increased airway smooth muscle and increased bronchodilator reversibility. Randomised controlled trials of vitamin D supplementation are warranted in paediatric STRA.

\section{S34 THE ADHESION RECEPTOR CADM1 ON MAST CELLS MEDIATES ADHESION TO LUNG FIBROBLASTS AND SMOOTH MUSCLE}

doi:10.1136/thoraxjnl-2011-201054b.34

E P Moiseeva, K Roach, M L Leyland, P Bradding. University of Leicester, Leicester, UK

Introduction and Objectives Cell adhesion molecule 1 (CADM1) contributes to cell-cell adhesion, viability and adhesion-induced degranulation in mast cells. We found that it is expressed as three alternatively spliced isoforms (major SP4, SP1 and minor SP6) in human lung mast cells (HLMCs). Here we investigated the role of CADM1 isoforms in the adhesion of mast cells to primary airway smooth muscle cells (ASM) and lung fibroblasts (LF).

Methods Modulation of CADM1 expression was investigated in transient transfection or adenoviral delivery in the mast cell line HMC-1 and HLMCs. Cells with overexpressed CADM1 isoforms or downregulated CADM1 were examined in cell adhesion assays.

Results CADM1 RNA interference in HMC-1 resulted in $60 \%$ reduction of surface CADM1 and complete loss of CADM1 determined by FACS and Western blotting, respectively, 6 days after transduction. This decrease in CADM1 expression reduced adhesion of transduced HMC-1 cells to ASM and LF by $42 \%$ and $50 \%$, respectively. Downregulation of CADM1 in HLMCs reduced adhesion to ASM by 39\%. HMC-1 cells transfected with SP1 (exon 8/9/ 11) and SP6 (exon 8/9/10/11) showed lower adhesion to LF compared to cells transfected with SP4 (exon 8/11). No differences in adhesion to ASM were found. When SP4 and SP1 isoforms were overexpressed up to $206 \%$ and $148 \%$ on the cell surface 6 days post transduction by viral delivery, this CADM1 overexpression did not change adhesion to ASM. However, increased levels of SP4 isoform increased mast cell adhesion to LF by $37 \%$. Thus, CADM1 isoforms affected adhesion to LF differently. The CADM1 counter-receptors, examined by Western blotting, are nectin-3 on ASM and CADM1 + nectin-3 on LF.

Conclusions CADM1 contributes significantly to mast cell adhesion to ASM and LF. Mast cell adhesion to ASM is likely to be limited by the number of counter-receptors on ASM. In contrast, mast cell adhesion to LF is determined by the levels of SP4 isoform on mast cells. Increased levels of other CADM1 isoforms do not increase adhesion to LF. It is likely that increased levels of longer SP1 and SP6 isoforms in proportion to the CADM1 pool decrease mast cell adhesion to LF.

\section{S35 RAPAMYCIN INHIBITS IL-33-INDUCED, NUOCYTE-DRIVEN AIRWAY INFLAMMATION}

doi:10.1136/thoraxjn--2011-201054b.35

A S Mirchandani, R J Salmond, C J Bain, F Y Liew. University of Glasgow, Glasgow, UK

Introduction IL-33 is an innate cytokine that promotes Th2 responses in both the innate and the adaptive immune systems, with an established role in allergic airway inflammation. ${ }^{1}$ The signalling pathway of IL-33/ ST2 is incompletely understood and the cells driving IL-33-mediated inflammation have remained elusive. Nuocytes, also known as natural helper cells, are a novel 
subpopulation of lineage negative innate cells that respond to IL-33 and IL-2. ${ }^{2}$ Rapamycin is a macrolide antibiotic that allosterically blocks mTOR, a serine-threonine kinase involved in numerous cellular signalling pathways.

Aim To determine the role of mTOR in IL-33-induced airway inflammation and the effect of rapamycin on IL-33-induced nuocytes in the lung.

Method BALB/c mice were treated with $1 \mu \mathrm{g}$ of IL-33 intranasally for 5 consecutive days in the presence or absence of rapamycin. Bronchoalveolar lavage (BAL) for cellular and cytokine analysis was performed. Fluorescence-activated cell sorting (FACS) of lung digests were analysed for intracellular IL-5 and cell surface markers.

Results IL-33 induced profound airway cellular infiltration noted in the BAL that was significantly inhibited by rapamycin. Cytokine levels from BAL fluid were also significantly reduced in mice treated with IL-33+ rapamycin. FACS analysis of lung digests demonstrated that IL-33 induced the expansion of lineage negative cells, in keeping with a population of nuocytes, which were the main source of IL-5 in the lung. Furthermore, this population of cells was suppressed by rapamycin.

Discussion Intranasal IL-33 drives mTOR-dependant airway inflammation. Nuocytes are the main source of IL-5 in IL-33-driven airway inflammation. Rapamycin inhibits the production of IL-5 and IL-13 in vivo as well as the expansion of nuocytes in the lung.

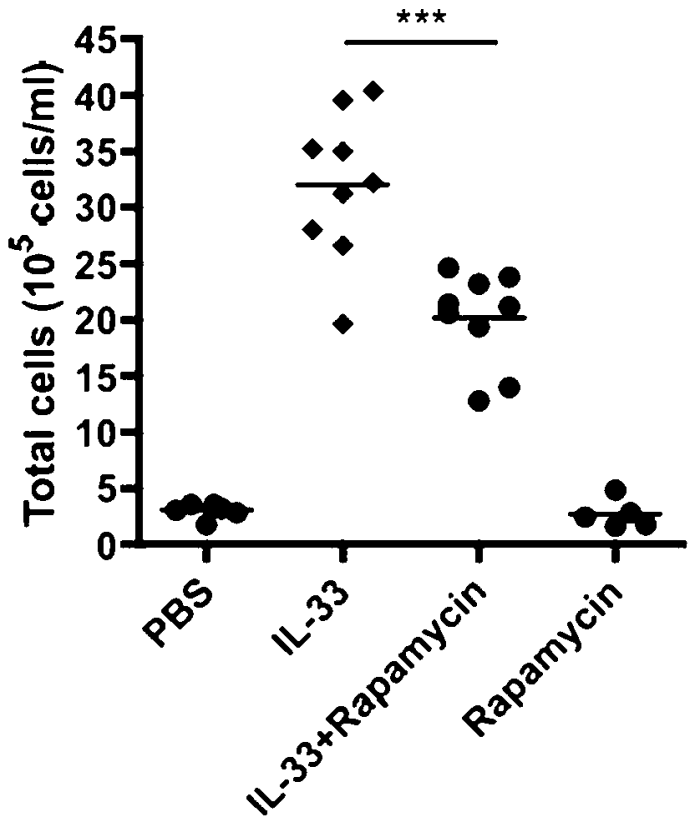

Abstract S35 Figure $1 \quad$ BALB/c mice were treated intranasally with $1 \mu \mathrm{g} \mathrm{IL}-33$ in the presence or absence of $1 \mathrm{mg} / \mathrm{kg}$ rapamycin for 5 consecutive days. The mice were sacrificed on day 6 and BAL total cell counts were performed. ${ }^{* *}=\mathrm{p}<0.001$.

\section{REFERENCES}

1. Lloyd CM. Curr Opin Immunol 2010;22:800-6.

2. Neill DR, et al. J Appl Physiol 2009;107:1258-65.

\section{S36 THE ADHESION RECEPTOR CADM1 PROMOTES HUMAN MAST CELL VIABILITY}

doi:10.1136/thoraxjnl-2011-201054b.36

E P Moiseeva, M L Leyland, P Bradding. University of Leicester, Leicester, UK

Introduction and objectives Cell adhesion molecule 1 (CADM1) contributes to cell-cell adhesion, proliferation and adhesion-induced degranulation in human lung mast cells. Previously we found that it is expressed as three alternatively spliced isoforms (major SP4 and SP1, and minor SP6) in human lung mast cells (HLMCs) and only SP4 in the cell line HMC-1, originating from mast cell leukaemia. Here we investigated the role of CADM1 in the viability of HLMCs and HMC-1.

Methods Modulation of CADM1 expression was investigated using adenoviral delivery in both HMC-1 and HLMCs. Cell viability in the absence of survival factors was determined by measuring cell numbers and caspase- $3 / 7$ activity.

Results Modulation of CADM1 expression in HMC-1 cells and HLMCs by overexpressing SP4 (exon 8/11) and SP1 (exon 8/9/11) and RNA interference was confirmed by FACS and Western blotting. Overexpression of SP4 did not affect HMC-1 viability $(105 \% \pm 7 \%$ of original number of cells) or caspase- $3 / 7$ activity $(7.7 \pm 0.2 \mathrm{FU} /$ cell $)$ in IMDM only for $48 \mathrm{~h}$ vs GFP-transduced cells, but overexpression of SP1 reduced cell number $(66 \% \pm 1 \%)$ and increased caspase- $3 / 7$ activity $(9.8 \pm 0.3 \mathrm{FU} / \mathrm{cell})$. CADM1 knockdown reduced HMC-1 number $(71 \% \pm 2 \%)$ and increased caspase- $3 / 7$ activity $(9.5 \pm 0.2 \mathrm{FU} /$ cell) in these conditions. In contrast to HMC-1 cells, overexpression of SP4 in HLMCs enhanced cell survival (39\% $\pm 3 \%$ ) in IMDM alone after $72 \mathrm{~h}$ compared to non-transduced cells $(31 \% \pm 1 \%$, whereas downregulation of CADM1 reduced cell number to $26 \% \pm 2 \%$. Caspase3/7 activity was increased in HLMCs with downregulated CADM1 $(99 \pm 20 \mathrm{FU} /$ cell) compared to SP4-overexpressing and nontransduced cells $(40 \pm 4 \mathrm{FU} /$ cell and $20 \pm 1 \mathrm{FU} /$ cell respectively). HLMCs displayed lower basal levels of Mcl-1, parallelled with lower survival and higher caspase-3/7 activation compared to HMC-1 cells. CADM1 downregulation in HLMCs coincided with decreased basal expression of Kit and Mcl-1.

Conclusions Modulation of CADM1 isoform expression or CADM1 downregulation in HMC-1 cells reduced survival. Conversely, SP4overexpression or CADM1 downregulation in HLMCs resulted in increased survival or increased cell death, respectively. CADM1 modulation in HLMCs coincided with modulation of proteins related to survival. Hence, CADM1 promotes survival in human mast cells.

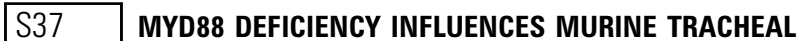 EPITHELIAL METAPLASIA AND SUBMUCOSAL GLAND ABUNDANCE}

doi:10.1136/thoraxjnl-2011-201054b.37

${ }^{1} A$ Giangreco, 'L Lu, 'D Mazzatti, ${ }^{2} B$ Spencer-Dene, ${ }^{2} E$ Nye, ${ }^{1} V$ Teixeira, ${ }^{1} S$ Janes. ${ }^{1}$ University College London, London, UK; ${ }^{2}$ Cancer Research UK, London, UK

Tracheal epithelial remodelling, excess mucus production, and submucosal gland hyperplasia are features of numerous lung diseases, yet their origins remain poorly understood. Previous studies have suggested that NF-?B signalling may regulate airway epithelial homeostasis. The purpose of this study was to determine whether deletion of the NF-?B signalling pathway protein myeloid differentiation factor 88 (Myd88) influenced tracheal epithelial cell phenotype. We compared wild-type and Myd88-deficient or pharmacologically inhibited adult mouse tracheas and determined that in vivo Myd88 deletion resulted in increased submucosal gland number, secretory cell metaplasia, and excess mucus cell abundance. We also found that Myd88 was required for normal resolution after acute tracheal epithelial injury. Microarray analysis revealed that uninjured Myd88-deficient tracheas contained 103 transcripts that were differentially expressed relative to wild-type and all injured whole tracheal samples. These clustered into several ontologies and networks that are known to functionally influence epithelial cell phenotype. Comparing these transcripts to those expressed in airway progenitor cells revealed only five common genes, suggesting that Myd88 influences tracheal epithelial homeostasis through an 